\title{
New Limits to the Infrared Background: Bounds on Radiative Neutrino Decay and on Contributions of Very Massive Objects to the Dark Matter Problem
}

\author{
S. D. Biller, ${ }^{1}$ J. Buckley, ${ }^{2}$ A. Burdett ${ }^{3}$ J. Bussons Gordo, ${ }^{4}$ D. A. Carter-Lewis, ${ }^{5}$ D. J. Fegan, ${ }^{4}$ J. Finley, ${ }^{6}$ J. A. Gaidos, ${ }^{6}$ \\ A. M. Hillas, ${ }^{3}$ F. Krennrich, ${ }^{5}$ R. C. Lamb,${ }^{7}$ R. Lessard,${ }^{6}$ J. E. McEnery, ${ }^{4}$ G. Mohanty, ${ }^{5}$ J. Quinn, ${ }^{4}$ A. J. Rodgers, ${ }^{3}$ \\ H. J. Rose, ${ }^{3}$ F. Samuelson, ${ }^{5}$ G. Sembroski, ${ }^{6}$ P. Skelton, ${ }^{3}$ T. C. Weekes,${ }^{8}$ and J. Zweerink ${ }^{5}$ \\ ${ }^{1}$ Oxford University, Oxford, United Kingdom \\ ${ }^{2}$ Washington University, St. Louis, Missouri 63130 \\ ${ }^{3}$ University of Leeds, Leeds, United Kingdom \\ ${ }^{4}$ University College, Dublin, Ireland \\ ${ }^{5}$ Iowa State University, Ames, Iowa 50011 \\ ${ }^{6}$ Purdue University, West Lafayette, Indiana 47907 \\ ${ }^{7}$ California Institute of Technology, Pasadena, California 91125 \\ ${ }^{8}$ Whipple Observatory, Amado, Arizona 85645 \\ (Received 19 September 1997; revised manuscript received 9 December 1997)
}

\begin{abstract}
From considering the effect of $\gamma-\gamma$ interactions on recently observed $\mathrm{TeV}$ gamma-ray spectra, improved limits are set to the density of extragalactic infrared photons which are robust and essentially model independent. The resulting limits are more than an order of magnitude more restrictive than direct observations in the $0.025-0.3 \mathrm{eV}$ regime. These limits are used to improve constraints on radiative neutrino decay in the mass range above $0.05 \mathrm{eV}$ and to rule out very massive objects as providing the dark matter needed to explain galaxy rotation curves. Lower bounds on the maximum distance which TeV gamma rays may probe are also derived. [S0031-9007(98)05639-7]
\end{abstract}

PACS numbers: $98.70 . \mathrm{Vc}, 13.35 . \mathrm{Hb}, 95.35 .+\mathrm{d}, 98.54 .-\mathrm{h}$

The extragalactic background infrared (IR) field contains a potential wealth of information relevant to both cosmology and particle physics (see, for example, [1]), but has so far eluded any conclusive detection. Direct measurement of the characteristics of this radiation are frustrated by the dominance of local, galactic IR sources. $\mathrm{TeV}$ gamma-ray astronomy provides a means to study the IR background indirectly, free of such complications, by looking for modifications to high energy gamma-ray spectra due to interactions with this background field via the process $\gamma \gamma \rightarrow e^{+} e^{-}$. This idea was first noted by Gould and Schréder [2] and has been more recently restated by Stecker and de Jager [3].

Previous IR studies making use of this phenomenon have generally used spectral data from the active galactic nucleus (AGN) Mrk 421 taken by the EGRET [4] and Whipple [5] experiments at $\mathrm{GeV}$ and $\mathrm{TeV}$ energies, respectively. These studies have relied on extrapolating flux measurements between these two energy regimes in an effort to determine the inherent shape of the source spectrum [3,6-8]. This approach has several difficulties: (1) Since the flux level is known to be highly variable at $\mathrm{TeV}$ energies, the relative normalization between EGRET and Whipple flux measurements is not known; (2) the flux of IR photons cannot be assumed to be zero in the intervening energy range; and (3) the shape of the inherent source spectrum is not known and cannot be assumed to necessarily follow a single power law from $\mathrm{GeV}$ to $\mathrm{TeV}$ energies. Indeed, current best fit values for Mrk 421 at $\mathrm{TeV}$ energies indicate a steeper spectrum than is reflected by the EGRET data [9], suggesting that the spectral shape changes in the intervening energy range. In addition, most of the analyses have assumed a particular model to describe the shape of the IR photon spectrum. Even a more recent attempt at a less model-dependent limit using data from Mrk 501 nevertheless assumes a power-law source spectrum, an arbitrary IR flux normalization, and constant IR energy densities over energy ranges spanning up to an order of magnitude in extent [10]. Limits derived in this manner may not apply if the background IR spectrum is of a different shape and, in particular, cannot necessarily be used to constrain mechanisms which might produce sharp features in the spectrum on a scale smaller than that of the assumed model. This paper will attempt to define more robust limits based on recent high energy observations of AGN which do not rely on extrapolation from lower energies or the assumption of a strict power-law source spectrum and are independent of the IR model down to scales corresponding to a factor of 2 change in IR energy. These limits will then be used to constrain significantly the possible contribution of very massive objects (VMOs) to the dark matter problem and also to place improved bounds on the radiative decay of massive neutrinos in the mass range above $\sim 0.05 \mathrm{eV}$, which is in the regime of interest to the atmospheric neutrino problem.

Derived upper limits to the background IR-Two active galaxies, Mrk 421 and Mrk 501, have thus far been seen to produce $\mathrm{TeV}$ gamma-ray emission by independent experimental groups. Both of these active galaxies are in the blazar class and are located at redshifts of 0.031 and 0.033 , respectively. Both objects have exhibited significant flux variability at $\mathrm{TeV}$ energies, changing by an 
order of magnitude or more with characteristic time scales of less than an hour (in the case of Mrk 421, as short as 15 minutes). Recent analyses of data from Mrk 421 by the Whipple group $[9,11]$ indicate a differential spectrum with an $\sim E^{-2.6}$ power law extending to energies beyond $5 \mathrm{TeV}$, possibly as high as $8 \mathrm{TeV}$. Similarly, the HEGRA collaboration has measured the "high-state" spectrum of Mrk 501 [12] and has found it to be described by an $\sim E^{-2.5}$ power law extending to energies as high as $10 \mathrm{TeV}$.

For the purposes of this paper, the available data on active galaxies at a redshift of $\sim 0.03$ will thus be taken to indicate a spectral shape which is bounded at the 90\% confidence level between the power laws $E^{-2.3}$ and $E^{-2.8}$ and normalized by an arbitrary flux constant. It will be assumed that the measured spectra continue up to energies of $10 \mathrm{TeV}$. However, to demonstrate the effect of possible systematic uncertainties in the energy scale, more conservative limits will also be computed assuming a maximum gamma-ray energy of $6 \mathrm{TeV}$. Finally, it will be assumed that the inherent differential source spectrum is steeper than an $E^{-2}$ power law, although the specific shape need not be a power law itself. The limits derived are relatively insensitive to this latter choice. For example, selecting $E^{-1.5}$ as a bound was found to change the inferred limits by less than $30 \%$.

For ease of computation, the high energy spectrum is taken to consist of differential flux measurements at the following discrete energies: $0.4,0.75,1.0,1.5,2.0,3.0$, 4.0, 6.0, and $10.0 \mathrm{TeV}$. The spectrum of IR photons is approximated by a series of separate intervals spanning the energies $0.025-0.042,0.042-0.083,0.083-0.167$, $0.167-0.33,0.33-0.625$, and $0.625-1.0 \mathrm{eV}$. Within each interval, the value of $\varepsilon^{2} n(\varepsilon)$ is taken as constant, where $\varepsilon$ is the energy of the IR photon and $n$ is the spectral density of photons [i.e., photons $/ \mathrm{cm}^{3} / \mathrm{eV}$ ]. The value of $\varepsilon^{2} n(\varepsilon)$ among these intervals can thus be varied to reflect different background IR models and the corresponding effect of $\gamma-\gamma$ absorption on $\mathrm{TeV}$ gamma rays calculated according to the prescription described by previous papers $[3,8]$. For these calculations, a conservative value for the Hubble constant of $85 \mathrm{~km} / \mathrm{s} / \mathrm{Mpc}$ is assumed [13].

To calculate upper limits, the inherent AGN differential spectrum was allowed to assume any shape steeper than $E^{-2}$, and the values of $\varepsilon^{2} n(\varepsilon)$ among IR intervals were allowed to take on any values subject to the following constraints: (1) that these values are lower than the directly determined upper bounds imposed by other experiments (shown in Fig. 1), and (2) that the attenuation implied by the IR model would not cause the shape of the observed $\mathrm{TeV}$ spectrum to deviate outside the power-law bounds previously defined based on the data. This approach essentially anchors the lower energy $\mathrm{TeV}$ data to the appropriate observational upper bounds and then extends these bounds based on the shape and extent of the AGN spectra at higher energies. From these considerations, the maximum value of energy density permitted in each IR interval was determined. The resulting limits are

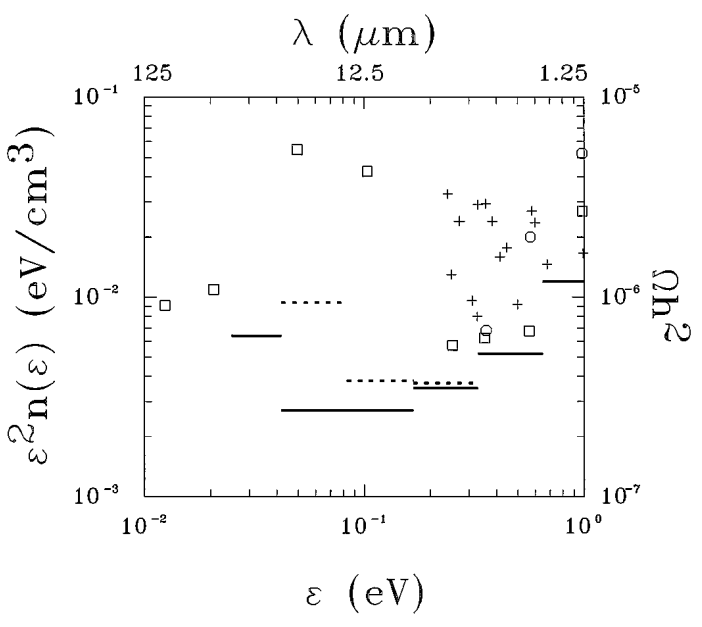

FIG. 1. Upper limits to the background infrared background: $(+)$ symbols are from Matsumoto et al. [14]; ( $\square$ ) from Hauser [15]; (O) from Kashlinsky et al. [16]. The solid lines show the limits derived in this work for a maximum gamma-ray energy of $10 \mathrm{TeV}$, while the dashed lines correspond to a maximum energy of $6 \mathrm{TeV}$.

summarized in Table I and are also plotted in Fig. 1 for assumed maximum gamma-ray energies of 6 and $10 \mathrm{TeV}$. Note that this choice of maximum energy affects primarily only the two lowest energy IR intervals.

In the range of $0.025-0.33 \mathrm{eV}$, the limits presented here are more than an order of magnitude more restrictive than those of DIRBE [15]. Note that these limits apply only to models which would produce features in the IR spectrum on a scale comparable to or larger than the interval widths used in the calculation. Monoenergetic line features, for example, are not necessarily constrained by these limits.

Constraints on VMO Abundance-Under certain cosmological scenarios, density fluctuations in the early universe at a redshift of $\sim 1000$ could give rise to the pregalactic formation of massive stars $\left(200<M / M_{\odot}<\right.$ $10^{5}$ ) at redshifts in the range of $\sim 100-300$ [17]. Such very massive objects would collapse to black holes after their main-sequence phase without any significant metal ejection. It has been suggested that the remnants of VMOs, which would ultimately cluster with galaxies, might provide an explanation for the dark matter associated with galaxy rotation curves $[18,19]$.

TABLE I. Derived upper limits to $\varepsilon^{2} n(\varepsilon)$.

\begin{tabular}{ccc}
\hline \hline & \multicolumn{3}{c}{ Upper limit to $\varepsilon^{2} n(\varepsilon)\left(\mathrm{eV} / \mathrm{cm}^{3}\right)$} \\
Energy interval $(\mathrm{eV})$ & $E_{\max }=10 \mathrm{TeV}$ & $E_{\max }=6 \mathrm{TeV}$ \\
\hline $0.025-0.042$ & 0.0064 & $\ldots$ \\
$0.042-0.083$ & 0.0027 & 0.094 \\
$0.083-0.167$ & 0.0027 & 0.0038 \\
$0.167-0.33$ & 0.0035 & 0.0037 \\
$0.33-0.625$ & 0.0052 & 0.0052 \\
$0.625-1.0$ & 0.012 & 0.012 \\
\hline \hline
\end{tabular}


While invisible now, VMOs would have produced a significant flux of IR photons during their burning phase. The peak number density of these photons is given [1] by

$$
\begin{gathered}
\varepsilon_{\mathrm{pk}}^{2} n_{V}\left(\varepsilon_{\mathrm{pk}}\right) \simeq 40 \frac{\Omega_{V} h^{2}}{1+z_{V}}, \\
\varepsilon_{\mathrm{pk}} \simeq \frac{30}{1+z_{V}},
\end{gathered}
$$

where $\varepsilon_{\mathrm{pk}}$ is the IR photon energy (in $\mathrm{eV}$ ) corresponding to the peak of the VMO contribution, $n_{V}$ is the photon spectral density due to VMOs in units of $\mathrm{cm}^{-3} \mathrm{eV}^{-1}$, $\Omega_{V}$ is the mass density of VMOs expressed as a fraction of the critical density, $z_{V}$ is the formation redshift of the VMOs, and the Hubble constant is given by $H_{0}=100 h(\mathrm{~km} / \mathrm{s}) / \mathrm{Mpc}$. Thus, the IR limits derived previously may be used to place upper bounds on $\Omega_{V}$. These bounds are shown in Fig. 2 as a function of the assumed VMO formation redshift for $h=0.65$ (which is conservative for this limit). Note that, for either maximum gamma-ray energy assumed, the limit on $\Omega_{V}$ is less than 0.065 for the redshift range of 100-300. Hence, unless substantial reprocessing of radiation by dust is assumed, these limits appear to rule out VMO models as providing the explanation for observed galaxy rotation curves.

Constraints on radiative neutrino decay-Several experiments have observed an apparent discrepancy in the ratio of $\nu_{e}$ to $\nu_{\mu}$ fluxes expected from cosmic ray interactions in the atmosphere [20-22]. A possible explanation for this effect may involve two-component neutrino oscillations with $\Delta m^{2} \sim 0.01 \mathrm{eV}^{2}$ and, thus, a neutrino mass in the regime of $0.1 \mathrm{eV}$ (provided the mass of the lighter neutrino is comparable to or smaller than this value). If neutrinos have mass, and different flavor eigenstates may mix, then massive neutrinos might decay via the process

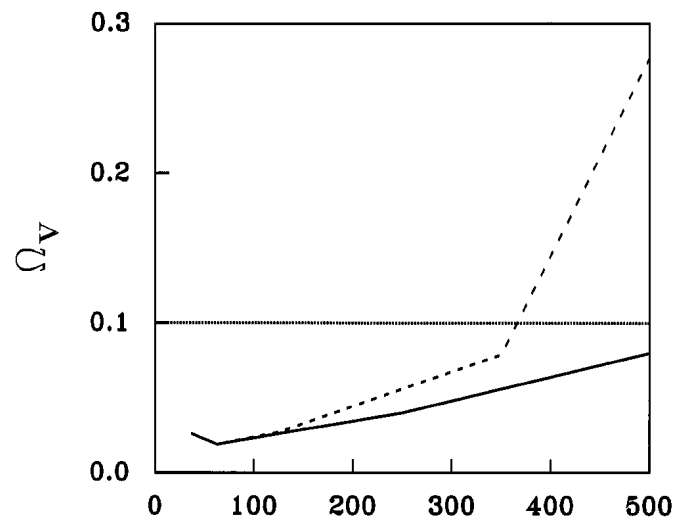

Formation Redshift

FIG. 2. Upper limits to the abundance of very massive objects based on bounds to the IR background derived in this work. The limit is expressed as a fraction of the critical density and is plotted as a function of the assumed formation redshift of the VMOs. The solid line corresponds to an assumed maximum gamma-ray energy of $10 \mathrm{TeV}$ and the dashed line to a maximum energy of $6 \mathrm{TeV}$.
$\nu_{H} \rightarrow \nu_{L}+\gamma$, where $m_{\nu_{H}}>m_{\nu_{L}}$. For neutrino masses in the $\sim \mathrm{eV}$ regime, such a radiative decay process would contribute to the infrared background. Although monoenergetic photons would be initially produced from a twobody decay, the photon spectrum would be broadened for relic neutrinos which decay over a range of redshifts such that the background density can be constrained by the limits presented previously.

Some unstable relic neutrinos with a lifetime longer than that of the universe would still be decaying now and would have produced a background photon density given by

$$
n_{\gamma}(\epsilon) \simeq 1.1 \times 10^{12} \frac{B_{r}}{\tau_{\nu} h \epsilon},
$$

where $\epsilon$ is half the rest mass of the unstable neutrino in $\mathrm{eV}, n_{\gamma}$ is the photon spectral density due to neutrino decay in units of $\mathrm{cm}^{-3} \mathrm{eV}^{-1}, B_{r}$ is the branching ratio for the radiative decay mode and $\tau_{\nu}$ is the neutrino lifetime in years [23]. Implied lower bounds for $\tau_{\nu} / B_{r}$ in the neutrino mass range $0.05<m_{\nu}<1 \mathrm{eV}$ are shown in Fig. 3 based on the IR limits presented in this paper for $h=0.85$. Previous constraints in this mass range from the work of Ressell and Turner [23] are shown for comparison. This represents an improvement of the constraints in some cases by more than 2 orders of magnitude.

Similarly, neutrinos with lifetimes shorter than the age of the universe but longer than the age at which decoupling of matter and radiation occurred will result in a background photon density given by

$$
n_{\gamma}(\epsilon) \simeq 115 \frac{B_{r}}{\epsilon},
$$

where, in this case,

$$
\epsilon=\left(\frac{\frac{1}{2} m_{\nu} c^{2}}{1 \mathrm{eV}}\right)\left(\frac{\tau_{\nu} \sqrt{\Omega_{0}} h}{7.4 \times 10^{9} \mathrm{yr}}\right)^{2 / 3},
$$

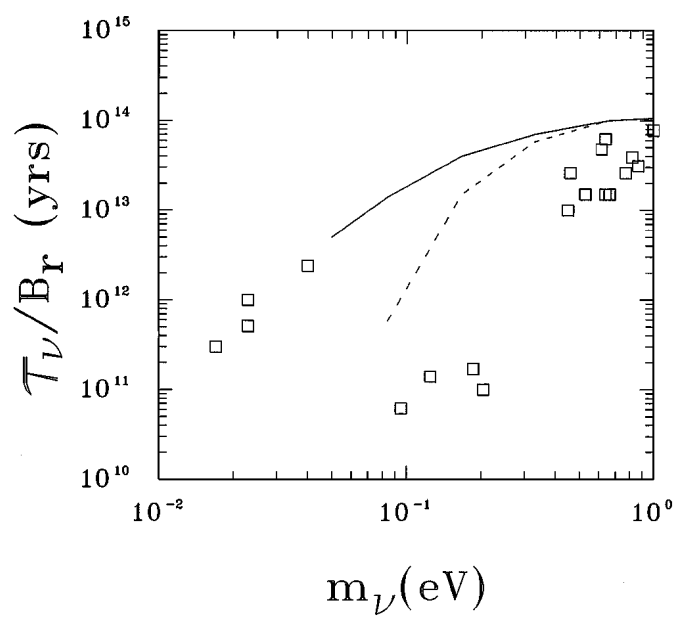

FIG. 3. Lower bounds to the ratio of the neutrino lifetime to the branching ratio for radiative decay based on this current work for the case where the neutrino lifetime is greater than the current age of the universe. The solid line shows the limits derived here assuming a maximum gamma-ray energy of $10 \mathrm{TeV}$, while the dashed line corresponds to a maximum energy of $6 \mathrm{TeV}$. ( $\square$ ) symbols show the limits derived previously by Ressell and Turner [23]. 


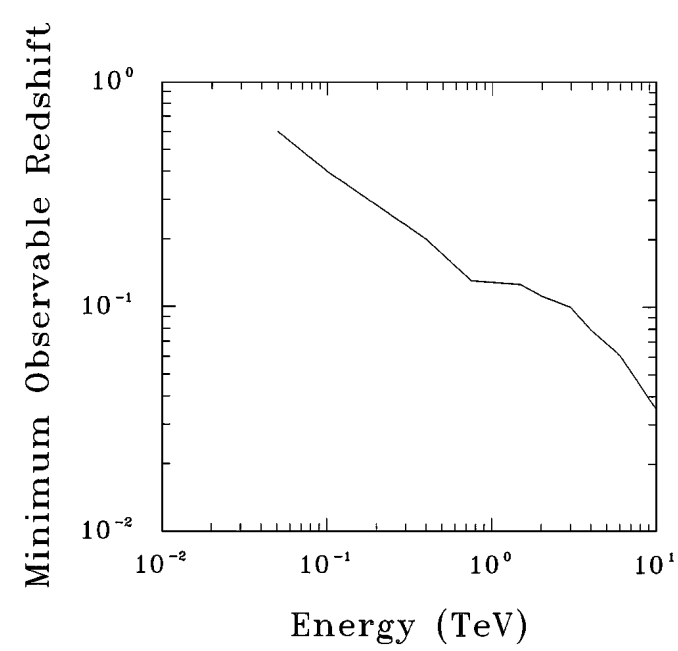

FIG. 4. Lower limits to the maximum redshift to which TeV gamma-ray sources are visible as a function of primary gammaray energy, based on limits to the background density of IR/UV photons.

where $\Omega_{0}$ is the mass density of the universe in units of the critical density. IR limits given here have thus been used to define exclusion contours which can be conveniently characterized by

$$
B_{r}<10^{-4}\left(\frac{1 \mathrm{eV}}{m_{0}}\right)
$$

for the region defined by

$$
m_{\nu}=\left(\frac{m_{0}}{1 \mathrm{eV}}\right)\left(\frac{7.4 \times 10^{9} \mathrm{yr}}{\tau_{\nu} \sqrt{\Omega_{0}} h}\right)^{2 / 3},
$$

where $0.05<m_{0}<2.0 \mathrm{eV}$ for an assumed maximum gamma-ray energy of $10 \mathrm{TeV}$, and $0.1<m_{0}<2.0 \mathrm{eV}$ for a maximum energy of $6 \mathrm{TeV}$. Once again, this represents an improvement in branching ratio sensitivity of approximately 2 orders of magnitude.

Substantial improvement in the ability to study the infrared background via $\gamma-\gamma$ interactions is expected with the next generation of instruments which will allow the behavior of more distant sources to be examined. In fact, the procedure used to derive the limits given in Table I may also be used to determine lower limits to the maximum distance to which $\mathrm{TeV}$ gamma-ray telescopes can be expected to probe. This has been done for the gamma-ray energy range of $0.4-10 \mathrm{TeV}$, assuming that a source remains "visible" out to an optical depth of 2 and that the majority of the IR was produced prior to the epoch of the sources under study. IR and UV upper bounds from direct observations in the energy range greater than $2 \mathrm{eV}$ have also been used to calculate this minimum distance for gamma-ray energies of 0.05 and $0.1 \mathrm{TeV}$. The results are shown in Fig. 4. The current generation of groundbased, gamma-ray telescopes have a typical lower energy threshold of $\sim 0.5 \mathrm{TeV}$ and are thus expected to be able to see sources possessing redshifts up to or beyond $z=0.1$. The next generation of instruments is expected to have an energy threshold in the region from 0.05 to $0.1 \mathrm{TeV}$, and will therefore be able to see out to a redshift of at least $z=0.5$. This represents a substantially larger volume of space than has so far been explored at $\mathrm{TeV}$ energies. This, and the known preponderance of AGN at larger redshifts, indicates a bright future for ground-based, gamma-ray astronomy and strongly suggests a drive towards higher duty cycles, larger apertures, and greater dynamic ranges for such instruments to explore this regime thoroughly.

This work has been supported in part by PPARC, Forbairt, the U.S. Department of Energy, NASA, and the Smithsonian Institution.

[1] J. R. Bond, B. J. Carr, and C. J. Hogan, Astrophys. J. 306, 428 (1986).

[2] R. P. Gould and G.P. Schréder, Phys. Rev. 155, 1408 (1967).

[3] F. W. Stecker and O. C. de Jager, Astrophys. J. Lett. 415, L71 (1993).

[4] R. C. Hartman et al., in Proceedings of the 2nd Compton Symposium, University of Maryland, 1994.

[5] G. Mohanty et al., in Proceedings of the 23rd I.C.R.C., Calgary, 1993.

[6] E. Dwek and J. Slavin, Astrophys. J. 436, 696 (1994).

[7] O. C. De Jager, F. W. Stecker, and M. H. Salamon, Nature (London) 369, 294 (1994).

[8] S. Biller et al., Astrophys. J. 445, 227 (1995).

[9] J. Zweerink et al., Astrophys. J. Lett. 490, L141 (1997).

[10] T. Stanev and A. Franceschini, Astrophys. J. 494, L159 (1998).

[11] F. Krennrich et al., Astrophys. J. 481, 758 (1997).

[12] F. Aharonian et al., Astron. Astrophys. 327, L5 (1997).

[13] R. M. Barnett et al., Phys. Rev. D 54, 1 (1996).

[14] T. Matsumoto, M. Akiba, and H. Murakami, Astrophys. J. 332, 575 (1988).

[15] M. G. Hauser, in Unveiling the Cosmic IR Background, edited by E. Dwek, AIP Conf. Proc. No. 348 (AIP, New York, 1996).

[16] A. Kashlinsky et al., Astrophys. J. 470, 681 (1996).

[17] J. R. Bond, W. D. Arnett, and B. J. Carr, Astrophys. J. 280, 825 (1984).

[18] B. J. Carr, J. R. Bond, and W. D. Arnett, Astrophys. J. 277, 445 (1984).

[19] S. M. Faber and J. S. Gallagher, Annu. Rev. Astron. Astrophys. 17, 135 (1979).

[20] R. Becker-Szendy et al., Phys. Rev. D 46, 3720 (1992).

[21] Y. Suzuki, in Proceedings of Neutrino '96, edited by K. Enquist, K. Huitu, and J. Maalompi (World Scientific, Singapore, 1996).

[22] W. W. M. Allison et al., Phys. Lett. B 391, 491 (1997).

[23] M. T. Ressell and M.S. Turner, Comments Astrophys., Comments Mod. Phys., C 14, No. 6, 323 (1990). 